\title{
No benefit of long-term oxygen therapy in moderate hypoxaemia in COPD
}

There are established benefits of 24-hour supplemental oxygen in patients with chronic obstructive pulmonary disease (COPD) and severe hypoxaemia having resting oxygen saturation $\leq 88 \%$ or $\mathrm{PO}_{2}$ $\leq 55 \mathrm{mmHg}(7.3 \mathrm{kPa})$, or $\leq 59 \mathrm{mmHg}(7.9 \mathrm{kPa})$ with signs of rightsided heart strain or polycythaemia. Supplemental oxygen has typically also been provided to the far larger group of people with normal resting oxygen levels that fall during exertion or persons of COPD with moderate resting hypoxaemia, but no benefits of this have ever been established.

The recent Long-Term Oxygen Treatment Trial (LOTT study), which was a USA-based multi-centre randomised trial published in the New England Journal of Medicine, is the largest study of longterm oxygen therapy to date. The LOTT study sought to address this practice and evaluate clinically relevant patient outcomes in daily life. ${ }^{[1]}$ The unblinded study was funded by the National Institutes of Health (USA) and recruited 738 participants with COPD (73\% males) at 42 centres who had either (i) mild-to-moderate hypoxaemia at rest (oxygen saturation of 89 - 93\%) or (ii) normal resting oxygen saturation that fell to 80 - 90\% during a 6-minute walk test. They were randomly assigned to receive either long-term supplemental oxygen or no oxygen. The supplemental oxygen was prescribed at $2 \mathrm{~L} \mathrm{O}_{2} /$ min continuously (at least 15 hours/day) in participants with resting hypoxaemia (57\%) and as an adjusted oxygen dose during exercise and $2 \mathrm{~L} \mathrm{O}_{2} /$ min during sleep in participants with exertional hypoxaemia only (43\%). They were followed for up to 6 years (median 1.5 years).
Patients receiving oxygen had no improvement in the primary outcome (rate of first hospitalisation or time to death after diagnosis, hazard ratio 0.94 ), quality of life, exercise capacity or COPD exacerbations compared with patients who did not receive supplemental oxygen. The authors concluded that 'among patients with COPD who have a resting $\mathrm{SpO}_{2}$ of $>88 \%$, long-term supplemental oxygen therapy does not result in longer survival than no oxygen therapy, regardless of whether the patients have exercise-induced desaturation.'

The study calls into question the current practice of routinely treating moderately hypoxaemic COPD patients with supplemental oxygen, and the tremendous expense incurred by state-funded institutions and private insurers.

This trial has far-reaching consequences for both the medical fraternity and funders in the practice of this often-used, expensive and unhelpful treatment, as this study showed.

\section{S Sinha Roy}

Pulmonology Fellow, Division of Pulmonology, Department of Medicine, Stellenbosch University and Tygerberg Academic Hospital, Cape Town, South Africa

1. The Long-Term Oxygen Treatment Trial Research Group, A randomized trial of longterm oxygen for COPD with moderate desaturation. N Engl J Med 2016;375(17):16171627. https://doi.org/10.1056/NEJMoa1604344

S Afr Respir J 2017;23(1):22. DOI:10.7196/SARJ.2017.v23i1.155 